\title{
Criterios de calidad del título de grado de fisioterapia. Informe de la Asociación Española de Fisioterapeutas
}

\author{
Quality criteria of physiotherapy grade (diploma). Report provided by the Spanish \\ Association and Physiotherapists
}

\author{
R. Fernández Cervantes, S. Souto Camba, J. Rebollo Roldán, A. Gómez Conesa, L. \\ González Doniz, M. Gutiérrez Nieto, P. Borrego Jiménez, J.M. Tricás Moreno, F.J. \\ Barbero Iglesias, M. López Viñas, E. Estébanez de Miguel
}

\begin{abstract}
Resumen
El informe sobre criterios de calidad específicos de recursos materiales y profesorado para el título de grado de Fisioterapia ha sido elaborado por la Asociación Española de Fisioterapeutas que convocó, con tal finalidad, a una comisión de expertos en Educación en Fisioterapia. El documento, realizado a propuesta de la Dirección General de Universidades en diciembre de 2004, tiene por objetivo el establecer los criterios mínimos de recursos materiales y humanos derivados de la especificidad de la titulación de Fisioterapia, que permita cubrir las nuevas demandas de la sociedad. En relación a los recursos materiales, se ha procurado darle un suficiente grado de especificación cuantitativa y cualitativa a los instrumentos que constituyen los recursos materiales específicos. La intención es, así mismo, facilitar su valoración en el momento de acreditar la titulación. Se abordan, por una parte, los recursos específicos de la titulación para la docencia teórico-práctica en el centro, dentro de los laboratorios destinados a tal efecto. Por otra parte, se definen las características o perfil de los centros sociosanitarios donde debe impartirse la formación clínica del alumno. En relación a los recursos humanos, se hace especial hincapié en el perfil y la experiencia del profesorado para la docencia, así como en el ratio profesor-alumno en la formación práctica y en la formación clínica.
\end{abstract}

\begin{abstract}
The report about specific quality criteria of resources material (items) and teaching profession to obtain the Physiotherapy Grade, has been elaborated by the Spanish Association of Physiotherapists, which aimed to convoke a Physiotherapy Education experts commission. The document was carried out on Universities General Chairmene's proposal, aiming to establish the minimum criteria of human and Material (Items) resources, derived from the specific Physical Therapy Grade (Diploma), Which may cover the latest Society requirements. According to the Material resources, there has been a serious attempt to give the instruments, which constitute the specific material resources, an appropriate degree, quantitative and qualitative specification. The main intention by itself, consists of providing its assessment when the time to accredit the title comes. On the one hand the specific resources of the Physiotherapy Grade for the Theoric-practical Teaching in the Centre are taken up even in the laboratories aimed to get such goal. On the other hand we define the characteristics or the profile of the social. Health centres, where Pupils' Clinical Education, must be carried out. Relating to the Human Resources, we do underline the Teaching Profession experience and profile, as well as the rate Teacher-Pupil, both in the practical Training and the Clinical Education, too.
\end{abstract}

Palabras clave

Calidad; Educación clínica; Fisioterapia.

Key words

Quality; Clinical education; Physical therapy. 


\section{Introducción}

A propuesta de la Dirección General de Universidades, en 2004, la Asociación Española de Fisioterapeutas (AEF) convocó a una comisión de expertos en Educación en Fisioterapia, para la elaboración de un informe relativo a los criterios de calidad del título de grado de fisioterapia. Una vez desarrollados dichos criterios, en marzo de 2005, se remitió a la Dirección General de Universidades el documento con las conclusiones de los "criterios de calidad específicos de recursos materiales y profesorado para título de grado de fisioterapia”. Este documento, se elaboró en atención a la solicitud formulada, a una delegación de la AEF el día 13 de diciembre de 2004, por doña Carmen García Rivas Directora General de Universidades en esa fecha.

El documento tiene por objetivo el establecer los criterios mínimos de recursos materiales y humanos derivados de la especificidad de la titulación de fisioterapia, que permita cubrir las nuevas demandas de la sociedad. El informe se ha elaborado con la intención de darle una amplitud de contenidos formativos necesarios para abordar las diferentes demandas de salud que presenta la sociedad, susceptibles de beneficiarse de la metodología terapéutica de la fisioterapia.

En relación a los recursos materiales, se ha procurado darle un suficiente grado de especificación cuantitativa y cualitativa a los instrumentos que constituyen los recursos materiales específicos. La intención es, así mismo, facilitar su valoración en el momento de acreditar la titulación.

Se abordan, por una parte, los recursos específicos de la titulación para la docencia teórico-práctica en el centro, dentro de los laboratorios destinados a tal efecto. Por otra parte, se definen las características o perfil de los centros sociosanitarios donde debe impartirse la formación clínica del alumno.

En relación a los recursos humanos, se hace especial hincapié en el perfil y la experiencia del profesorado para la docencia, así como en el ratio profesor-alumno en la formación práctica y en la formación clínica.

\section{Criterios de calidad en recursos materiales}

\section{Recursos específicos de la titulación para la docencia teórico-práctica en el centro: los laboratorios}

\section{Laboratorio de anatomía}

Se considera que la formación en Anatomía resulta fundamental en la formación de fisioterapeutas, por lo que sus prácticas deben basarse en la disección cadavérica.

Las necesidades mínimas de equipamiento deben permitir dotar una sala de disección de acuerdo a los criterios establecidos por el Acta de Barcelona de la Sociedad Anatómica Española, en su I Simposio sobre instalaciones y entorno de una sala de disección modelo, celebrado en Barcelona el 22 de junio de $1996^{1}$ :

Diseño de la sala de disección. Desde el punto de vista general en la sala de disección se deberían contemplar dos grandes áreas de trabajo, la dedicada a la docencia e investigación y la dedicada a la recepción y almacenamiento de cadáveres.

1. En el área dedicada a la docencia e investigación se localizarán las mesas para el estudio del cadáver y los servicios específicos como: modelos anatómicos, huesos, proyecciones, vídeos, radiografías, etc.

2. En el área docente debería existir una zona para cambiarse de ropa y lavamanos quirúrgicos.

3. En el área de recepción y almacenamiento, comunicada con la anterior, pero de uso exclusivo para el personal técnico, se encontrarían: a) un local específico para la recepción y embalsamamiento del cadáver; $b$ ) un local específico para tratamientos diversos (sierra, microdisección, técnicas de corrosión, plastinación, taller de montaje de esqueletos, recuperación de huesos, etc.); c) un local de depósito de piezas pequeñas y otro para cadáveres, y $d$ ) un almacén para materiales y sustancias tóxicas e inflamables, que reúna las condiciones exigidas por ley.

Se recomienda, que el área docente y de recepción-almacenamiento sean independientes entre ellas y con sistemas de seguridad para su acceso.

El área de recepción debería tener una entrada desde el exterior del edificio para facilitar el acceso de los cuerpos donados y la salida de restos, así como para la entrada y salida de materiales y/o sustancias químicas sin pasar por las áreas docentes. 


\section{Laboratorio de función del cuerpo humano}

Este laboratorio debe permitir la formación práctica de parte de los contenidos de las ciencias básicas, concretamente los referidos a la fisiología y la bioquímica. El equipamiento básico que deberá incluir este laboratorio es:

- Laboratorio virtual de fisiología para estudiantes en versión avanzada, equipado con un software y un hardware que permita las funciones de electrocardiografía, electroencefalograma, volumen pulmonar, esfuerzo respiratorio, etc.

- Balanza.

- pHmetro. • Espectrofotómetro (colorímetro).

- Centrífuga de mesa y una centrífuga refrigerada con diversos rotores.

- Placa de agitación magnética con calefacción.

- Agitador de tubos tipo Vortex.

- Baño de agua termostatizado.

- Fuente de alimentación y cubeta de electroforesis.

- Sistema para cromatografía en columna.

- Autoclave.

- Estufa de cultivo.

- Cajas con modelos moleculares.

- Sistema de filtración a vacío.

- Material de vidrio y plástico diversos (pipetas, vasos precipitados, erlenmeyers, probetas, tubos de ensayo, gradillas, etc.)

\section{Laboratorio de biomecánica y física aplicada}

Este laboratorio debe de permitir la formación práctica de los contenidos referidos a la biomecánica y física aplicada. El material o el equipamiento básico de este laboratorio deberá incluir los siguientes elementos:

Para los contenidos relacionados con la biomecánica:

- Cronómetro.

- Antropómetro.

- Compás deslizante o de corredera.

- Cinta antropométrica.

- Pesa.

- Instrumento de evaluación del arco de movimiento cervical.

- Equipo de evaluación postural.

- Podómetro.

- Medidor de escoliosis.

- Equipo de evaluación isocinética de la función muscular.

Para los contenidos relacionados con la física aplicada:

- Termómetros.

- Destilador de agua.

- Balanza de décimas de gramo.

- Veinte tubos de vidrio.

- Pignómetro.

- Dos viscosímetros.

- Estalagmómetro.

- Circuitos de corriente y de inducción electromagnética, que incluyan: 2 bobinas de 1.800 espiras, 2 bobinas de 400 espiras, 2 imanes rectos, 2 imanes de herradura, cables de conexión, resistencias de 1, 2,7, 4,7 y 12 kilohomios, 2 baterías de 9 V y 2 de 4,5 V, 2 condensadores y 4 multímetros.

- Material de protección radiológica y radiología que incluya: monitor de radiación, tubo didáctico de rayos $\mathrm{X}$, placas de dentista, 4 negatoscopios de cuatro y un negatoscopio vertical. 


\section{Laboratorios de fisioterapia}

La siguiente dotación pretende dar respuesta a la formación práctica de los siguientes contenidos formativos: valoración en fisioterapia, cinesiterapia, procedimientos generales de intervención en fisioterapia, métodos específicos de intervención en fisioterapia y fisioterapia en especialidades clínicas ${ }^{2-}$ 4

El material o el equipamiento básico de estos laboratorios deberá incluir los elementos mostrados en la tabla 1.

Se considera que para un lógico funcionamiento, estos recursos académicos deben estar distribuidos al menos en cuatro espacios diferenciados, referidos a los siguientes ámbitos:

- Hidroterapia.

- Electroterapia.

- Métodos generales de fisioterapia.

- Métodos específicos de fisioterapia.

Tabla 1. Material o equipamiento básico de los laboratorios de fisioterapia

\section{Material general de valoración}

2 espalderas de un cuerpo en madera

2 espejos de corrección cuadriculados de pared

110 medidores de sensibilidad Watemberg

5 aestesiómetros

15 goniómetros digitales (longitud del brazo $11 \mathrm{~cm}$ y longitud del brazo articulado $3 \mathrm{~cm}$ )

5 plomadas con cordón

5 medidores de presión arterial con brazalete de velcro

10 estetoscopios

15 goniómetros de Moeltgen en acero inoxidable

50 lápices dermatográficos rojo y negro

1 negatoscopio mural de un cuerpo

10 martillos de reflejos

10 medidores de pliegue cutáneo

1 pelvímetro

\section{Material general de tratamiento}

6 camillas hidráulicas articuladas (por laboratorio, para 10 alumnos)

6 camillas neurológicas

11 colchonetas

1 banco de cuádriceps y botas de Delorme

Sistemas de tracción cervical y lumbar

Sistemas de suspensión y poleoterapia

Rueda de hombro, material de propiocepción

Juego de halteras

Barras y balones medicinales

Balones de Bobath

Material general de laboratorio (almohadillas, cinchas, cuñas, rodillos)

\section{Material especial de valoración y tratamiento}

Equipos de onda corta y microonda con sus respectivos accesorios

Equipo de electroestimulación de baja y media frecuencia y accesorios

Equipo de estimulación eléctrica transcutánea (TENS)

Equipo de ultrasonidos y accesorios

Equipos de laserterapia y magnetoterapia

Equipos de termoterapia y crioterapia

Lámparas de infrarrojos y ultravioletas

Equipo de electromiofeedback: electromiógrafo de superficie y uroginecológico

Ecógrafo

Termógrafo

Equipamiento para la realización de ortesis

Material de valoración y tratamiento en fisioterapia

respiratoria

Espirómetro

Pulsioxímetro

Capnógrafo

Manómetro determinador de presiones en boca

Equipos de vibración endógena con presión positiva

oscilatoria

Espirometría incentivada volumétrica

Equipo de entrenamiento específico de la musculatura respiratoria

Sistema de presión espiratoria positiva

Tapiz rodante y bicicleta ergométrica

Sistema de esterilización

Material específico de hidroterapia

Tanque de Hubbar

Equipo para baños de contraste en miembros inferiores y

superiores

Ducha de Kneipp 
Recursos específicos de la titulación para la docencia clínica en los centros sociosanitarios: estancias clínicas

Las estancias clínicas constituyen un elemento indispensable dentro del proceso formativo del fisioterapeuta, al permitirle realizar el contacto directo con el medio asistencial y con el paciente, al mismo tiempo que se familiariza con el proceso de atención fisioterápica y su papel dentro del equipo multidisciplinar ${ }^{5-8}$.

El objetivo principal de la enseñanza clínica es el garantizar que los discentes adquieran la necesaria competencia clínica, entendiendo como tal, la capacidad cualificada para ejecutar adecuadamente las tareas clínicas propias del fisioterapeuta. La competencia clínica se relaciona con la capacidad de llevar a cabo las tareas profesionales en condiciones reales. Las tareas clínicas que se realizan en el seno de las clases clínicas son: el examen, la evaluación, el diagnóstico de fisioterapia, el pronóstico, la intervención en fisioterapia según cada patología, además de la prevención de la enfermedad, promoción de la salud y tareas organizativas.

Para garantizar este objetivo, las universidades que impartan el título de grado deben establecer los oportunos convenios con centros sociosanitarios de titularidad pública y/o privada que permitan encuadrar al alumnado garantizando que el proceso formativo sea cuantitativa y cualitativamente óptimo ${ }^{2,9}$. De tal modo que los diferentes centros permitan disponer de una oferta en la que se puedan desarrollar los diferentes contenidos temáticos que la formación de un fisioterapeuta requiere, entre otros, fisioterapia en envejecimiento, en atención primaria, en las algias vertebrales, en las patologías neurológicas y cardiorrespiratorias, fisioterapia en la incontinencia urinaria, etc., prestando especial atención al concepto de discapacidad en cada uno de los contenidos clínicos. En general, esta diversidad temática es difícil encontrarla en un solo centro sanitario, ni siquiera en los que cuentan con servicios de fisioterapia y rehabilitación más grandes.

Por otro lado, la formación clínica del alumnado debe ser impartida por profesorado clínico competente en su campo temático, evitando que los alumnos accedan a los centros de formación clínica sin que exista un riguroso control de los contenidos y actividades que han de llevar a cabo para su formación ${ }^{10}$.

\section{Criterios de calidad del profesorado del área de fisioterapia para impartir en el título de grado}

Debido al requerimiento del dominio clínico que han de adquirir los alumnos de fisioterapia en su campo, la formación de los contenidos específicos del área de fisioterapia deberá ser impartida por los profesores que posean la capacidad legal del ejercicio profesional de la misma. Todo ello con el objetivo de garantizar un perfil formativo propio que permita al alumno la adquisición de los conocimientos, las habilidades, destrezas y aptitudes propias de la disciplina.

El profesorado del área de fisioterapia ha de acreditar poseer un perfil profesional o investigador relacionado con los contenidos formativos que va a impartir.

La docencia clínica será impartida por un profesor necesariamente vinculado o perteneciente a la universidad a través de las correspondientes figuras de profesor recogidas en la legislación vigente. De especial interés son para esta disciplina las figuras de profesor vinculado o profesor asociado en ciencias de la salud. En cualquier caso el profesorado clínico debe poseer algún tipo de relación contractual con la universidad, que permita a los centros y departamentos desarrollar una correcta gestión del proceso formativo.

Para impartir docencia clínica, los profesores habrán de acreditar experiencia profesional superior a 3 años en el ámbito clínico en el que los alumnos efectuarán las prácticas. Así mismo, el profesorado clínico ha de adquirir formación en metodología docente que le permita establecer los objetivos, seleccionar metodolologías y planificar evaluaciones que garanticen un adecuado diseño y aplicación del proceso de enseñanza aprendizaje.

El ratio profesor/alumno en estancias clínicas será de 1 profesor/2 alumnos. El ratio profesor/alumno en los laboratorios de fisioterapia del centro será de 1/10 máximo.

\section{Conclusiones}

Los centros deberán proporcionar los recursos humanos y materiales suficientes, tanto para las materias comunes en los estudios de grado de fisioterapia, como para la optatividad y configuración curricular que establezca cada universidad que imparta la titulación. 
El profesorado de la titulación, tanto del área de fisioterapia como el de otras áreas de conocimiento, ha de acreditar poseer un perfil profesional o investigador relacionado con los contenidos formativos que va a impartir.

Las universidades que impartan el título de grado, deben establecer los oportunos convenios con centros sociosanitarios de titularidad pública o privada, que permitan encuadrar al alumnado y garantizar que el proceso formativo resultante sea cuantitativa y cualitativamente óptimo.

En cuanto a la formación clínica del estudiante, debe ser impartida por profesorado clínico competente en su campo temático dentro de la fisioterapia.

\section{Bibliografía}

1. Acta de Barcelona de la Sociedad Anatómica Española. I Simposio. Barcelona, 1996.

2. Libro blanco del Título de Grado en Fisioterapia. Agencia nacional de Evaluación de la Calidad y Acreditación, 2004.

3. Guía académica y programa de estudios de la Universidad Libre de Bruselas (Bélgica).

4. Guía académica y programa de estudios de la Hoogschool van Amsterdam (Holanda).

5. Documento European Physiotherapy Benchmark Statement, elaborado por la Región Europea de la World Confederation for Physical Therapy.

6. Real Decreto 1001/2002, de 27 de septiembre (BOE de 9 de octubre), por el que se establecen los Estatutos de Consejo General de Fisioterapeutas de España.

7. Documento de la Conserjería de Salud de la Junta de Andalucía: Gestión por competencias.

8. Documento de la European Network Physiotherapy in Higher Education Competence for a Physical Therapy Promgarm.

9. Cervantes R, Doniz L, Rodríguez B, Souto S. Libro del 10. aniversario de la E.U. de Fisioterapia de A Coruña, 1990-2001. A Coruña: Escuela Universitaria de Fisioterapia de A Coruña.

10. Actas y conclusiones de las II Jornadas Interuniversitarias del Área de Fisioterapia sobre la estancia clínica. En: III Jornadas Interuniversitarias del Área de Fisioterapia. Universidad e Extremadura. Badajoz, 23 y 24 de septiembre de 2004. 